\title{
Association between mannose-binding lectin, high-sensitivity $C$-reactive protein and the progression of diabetic nephropathy in type 1 diabetes
}

\author{
T. K. Hansen • C. Forsblom • M. Saraheimo • L. Thorn • \\ J. Wadén • P. Høyem • J. Østergaard • A. Flyvbjerg • \\ P.-H. Groop • on behalf of the FinnDiane Study Group
}

Received: 10 September 2009 / Accepted: 17 February 2010/Published online: 16 April 2010

(C) Springer-Verlag 2010

\begin{abstract}
Aims/hypothesis Diabetic nephropathy has been associated with low-grade inflammation and activation of the complement system in cross-sectional studies. Data from prospective studies are sparse. We investigated the associations of the complement activator mannose-binding lectin (MBL) and the inflammatory marker high-sensitivity C-reactive protein (hsCRP) with the development of nephropathy in a large prospective study of patients with type 1 diabetes from the Finnish Diabetic Nephropathy (FinnDiane) Study. Methods Baseline MBL and hsCRP were measured in 1,564 type 1 diabetes patients from the FinnDiane study, of whom 1,010 had a normal albumin excretion rate, 236
\end{abstract}

T. K. Hansen and C. Forsblom contributed equally to this study.

Electronic supplementary material The online version of this article (doi:10.1007/s00125-010-1742-8) contains supplementary material, which is available to authorised users.

T. K. Hansen $(\bowtie) \cdot$ P. Høyem · J. Østergaard · A. Flyvbjerg Immunoendocrine Research Unit, Department of Endocrinology and Internal Medicine and Medical Research Laboratories,

Clinical Institute, Aarhus University Hospital,

Norrebrogade 42-44,

DK-8000 Aarhus C, Denmark

e-mail: tkh@dadlnet.dk

C. Forsblom $\cdot$ M. Saraheimo $\cdot$ L. Thorn $\cdot$ J. Wadén •

P.-H. Groop $(\square)$

Folkhälsan Institute of Genetics, Folkhälsan Research Center,

Biomedicum Helsinki, University of Helsinki,

PO Box 63, FIN-00014 Helsinki, Finland

e-mail: per-henrik.groop@helsinki.fi

C. Forsblom • M. Saraheimo $\cdot$ L. Thorn · J. Wadén · P.-H. Groop

Department of Medicine, Division of Nephrology,

Helsinki University Central Hospital,

Helsinki, Finland had microalbuminuria and 318 had macroalbuminuria. The main outcome was progression in renal disease during follow-up.

Results Both baseline MBL $(p=0.038)$ and hsCRP $(p<0.001)$ increased with increasing level of albuminuria. During $5.8 \pm$ 2.2 years of follow-up, progression to a higher albuminuria level or end-stage renal disease (ESRD) occurred in 201 patients. MBL levels were higher in progressors compared with non-progressors at all steps of progression, and in a covariate adjusted multivariate Cox-regression analysis MBL levels above the median were significantly associated with progression from macroalbuminuria to ESRD (hazard ratio $1.88,95 \%$ CI $1.06-3.32, p=0.030$ ). In a univariate analysis, hsCRP levels above the median were significantly associated with progression from normal albumin excretion rate to microalbuminuria, but the association was only borderline significant after adjustment for covariates (hazard ratio $1.56,95 \%$ CI $0.97-2.51, p=0.068$ ).

Conclusions/interpretation This study demonstrates that concentrations of both MBL and hsCRP are associated with the progression of renal disease in type 1 diabetes.

Keywords Complement system · Complications · Diabetes · Immune system - Mannose-binding lectin . Nephropathy

\begin{tabular}{ll}
\multicolumn{2}{l}{ Abbreviations } \\
AER & Albumin excretion rate \\
ESRD & End-stage renal disease \\
FinnDiane & Finnish Diabetic Nephropathy Study \\
hsCRP & High-sensitivity C-reactive protein \\
MBL & Mannose-binding lectin \\
HR & Hazard ratio
\end{tabular}

Abbreviations

AER Albumin excretion rate

ESRD End-stage renal disease

FinnDiane Finnish Diabetic Nephropathy Study

hsCRP High-sensitivity C-reactive protein

HR Hazard ratio 


\section{Introduction}

Despite significant improvements in diabetes care with intensified treatment of hyperglycaemia and hypertension, a significant proportion of patients with type 1 diabetes still develop diabetic nephropathy [1]. It is generally believed that the pathogenesis of diabetic renal disease is multifactorial, and progression may involve both low-grade inflammation and activation of the complement system.

The complement system is an enzyme cascade of the innate immune system that is activated via one of three different pathways: the classical, the alternative and the mannose-binding lectin (MBL) pathways [2, 3]. The system has evolved as a defence against pathogens, but it may act as a double-edged sword and cause self-damage following adverse activation. This happens through deposition of the membrane attack complex (MAC, also called C5b-9), the cytolytic endproduct of the complement cascade, or through increased inflammation via release of the anaphylatoxins $\mathrm{C} 5 \mathrm{a}$ and $\mathrm{C} 3 \mathrm{a}$. Increased deposition of MAC is found in diabetic kidneys [4], and recent experimental data indicate that glycoxidation-mediated local complement activation may be a significant contributor to the progression of diabetic nephropathy [5]. This finding thus provides a possible link between hyperglycaemia and progression of diabetic kidney disease.

Animal studies have shown that $M B L 2$ knockout mice that are unable to activate the MBL pathway of the complement system are protected from the detrimental effects of diabetes on the kidneys [6], indicating that the MBL pathway plays a significant role in diabetic renal changes. In humans, MBL levels vary significantly from person to person because of frequently occurring polymorphisms within exon 1 as well as in the promoter region of the $M B L 2$ gene on chromosome 10 [7, 8]. The MBL2 gene is not a type 1 diabetes susceptibility gene per se [9], but patients with type 1 diabetes have significantly increased serum levels of $\mathrm{MBL}[10,11]$, and both high circulating levels of MBL and high-coding MBL genotypes are associated with the presence and development of microalbuminuria and diabetic nephropathy [12-14].

There is evidence of increased inflammatory activity in type 1 diabetes $[15,16]$. Markers of inflammation such as high sensitivity C-reactive protein (hsCRP) have been cross-sectionally associated with diabetic nephropathy in some studies [17], while in the DCCT/Epidemiology Of Diabetes Interventions And Complications (EDIC) study CRP levels were not associated with complications [16]. The relationship between CRP levels and glycaemic control is complex, and CRP levels may even increase with intensified treatment depending on the degree of weight gain associated with the treatment [18]. Circulating hsCRP levels may predict the development of albuminuria in longitudinal studies of patients with type 2 diabetes [19, 20]. Whether this is also the case in type 1 diabetes remains unclear, although there was no clear association between CRP level and the progression of nephropathy in the DCCT trial [21]. Circulating levels of MBL in patients with type 2 diabetes are not different from those of healthy people, but MBL alone and especially in combination with hsCRP provides prognostic information on the development of albuminuria during the long-term follow-up of normoalbuminuric patients with type 2 diabetes [22]. In the present study we investigated the association of the prevalence and incidence of diabetic nephropathy with MBL and hsCRP levels at baseline in a large prospective study of patients with type 1 diabetes from the Finnish Diabetic Nephropathy Study (FinnDiane).

\section{Methods}

Study design This study was part of the ongoing prospective FinnDiane Study, which is a nationwide, comprehensive multicentre study with the aim of identifying genetic and environmental risk factors for diabetic nephropathy in type 1 diabetes patients.

At baseline, patients underwent a thorough clinical investigation at a regular visit to the attending physician. The prospective study started in 2005 . For all patients in the present analysis, all available medical files, including laboratory data, were reviewed and changes in renal status were verified.

Study participants Out of an original cohort of 1,691 patients with data available on both serum CRP and MBL, 127 with CRP above $10 \mathrm{mg} / \mathrm{l}$ were excluded because of potential ongoing infection. Therefore, 1,564 patients participated in the present study and were followed for an average of $5.8 \pm 2.2$ years. Type 1 diabetes was defined as onset of diabetes before the age of 35 years and permanent insulin treatment initiated within 1 year of diagnosis.

The cohort was subdivided into 1010 patients with normal urinary albumin excretion rate (normoalbuminuria; albumin excretion rate [AER] $<20 \mu \mathrm{g} / \mathrm{min}$ or $<30 \mathrm{mg} / 24 \mathrm{~h}$ ), 236 patients with microalbuminuria $(20 \leq \mathrm{AER} \leq 200 \mu \mathrm{g} / \mathrm{min}$ or $30<$ AER $<300 \mathrm{mg} / 24 \mathrm{~h}$ ) and 318 patients with macroalbuminuria (AER $\geq 200 \mu \mathrm{g} / \mathrm{min}$ or $\geq 300 \mathrm{mg} / 24 \mathrm{~h}$ ), based on fulfilment of the criteria for AER in at least two out of three consecutive overnight or $24 \mathrm{~h}$ urine collections at baseline. All urine samples were screened with a urine stick to exclude any urinary tract infection. Patients with end-stage renal disease (ESRD), defined as patients on dialysis or having received a kidney transplant, were excluded.

Progression of renal disease was defined as follows. All local AER data between baseline and the follow-up visit 
were reviewed. Based on the AER in any two out of three consecutive urine collections during the follow-up period, the renal status of patients was classified as in the baseline examination. Progression was defined as a change from one level to a higher level of albuminuria or the development of ESRD. Patients without progression of renal disease and patients who regressed to a lower level of albuminuria were classified as non-progressors.

The ethics committees of all participating centres approved the study protocol. Written informed consent was obtained from each patient and the study was performed in accordance with the Declaration of Helsinki as revised in 2000 .

Measurements At the regular patient visits, data on medication and diabetic complications were recorded using a standardised questionnaire, which was completed by the patient's attending physician based on medical files. Blood pressure was measured twice in the sitting position with a mercury sphygmomanometer after a $10 \mathrm{~min}$ rest and the average of these measurements was used in the analysis. Height, weight and WHR were recorded, and blood was drawn for the measurement of $\mathrm{HbA}_{1 \mathrm{c}}$, lipids, creatinine, hsCRP and MBL.

$\mathrm{HbA}_{1 \mathrm{c}}$ was determined by standardised assays at each centre. Serum lipid and lipoprotein concentrations were measured by automated enzymatic methods using a Cobas Mira analyser (Hoffmann-La Roche, Basle, Switzerland). Serum creatinine was assessed by enzymatic methods at a central laboratory. AER was determined in one $24 \mathrm{~h}$ urine collection at the central laboratory by radioimmunoassay (Pharmacia, Uppsala, Sweden) or immunoturbidimetry. The serum concentration of MBL was determined using an inhouse time-resolved immunofluorometric assay [10], and hsCRP was measured by a photometric, immunochemical method with an ultrasensitive kit (Orion Diagnostica, Espoo, Finland). The within- and between-assay CVs were $4.0 \%$ and $6.5 \%$, respectively.

Statistical analysis Data are expressed as mean \pm SD for normally distributed values and as median with interquartile range for non-normally distributed values. Differences between groups for normally distributed variables were tested using ANOVA and non-parametric data with the Kruskal-Wallis test. Categorical variables were analysed with the $\chi^{2}$ test. Risk factors for the progression of diabetic nephropathy were assessed using Cox regression analysis. In the normoalbuminuric group, with the observed number of progressors, the study had an $80 \%$ power with an alpha level of 0.05 to detect a difference in CRP or MBL of 0.33 SDs. The corresponding differences for patients with microalbuminuria and macroalbuminuria at baseline were 0.52 and $0.36 \mathrm{SD}$. All calculations were performed with SPSS 15.0.1 (SPSS, Chicago, IL, USA).

\section{Results}

Baseline values Clinical characteristics of the patients grouped by albuminuria status are presented in Table 1 . There was a stepwise increase in median serum MBL levels with increasing albuminuria level (overall $p=0.038$ ), and patients with macroalbuminuria had significantly higher levels of MBL than patients with normoalbuminuria. Concentrations of hsCRP were also increased in patients with albuminuria at baseline (overall $p<0.001$ ), but MBL and hsCRP levels did not correlate $(r=0.015, p=0.56)$. Median MBL levels were slightly higher in men $(1864 \mu \mathrm{g} / \mathrm{l}$, IQR 634-3100) than in women $(1,643 \mu \mathrm{g} / \mathrm{l}$, IQR 505 2,$947 ; p=0.029$ ), while hsCRP concentrations were significantly higher among women $(2.10 \mathrm{mg} / \mathrm{l}$, IQR $1.41-3.62)$ than among men $(1.72 \mathrm{mg} / \mathrm{l}$, IQR $1.07-3.09, p<0.001)$. The frequency of use of non-steroidal anti-inflammatory agents (NSAIDs) was $3.9 \%, 6.0 \%$ and $15.3 \%$, and the use of statins $3.5 \%, 7.9 \%$ and $21.8 \%$ in patients with normal AER, microalbuminuria and macroalbuminuria, respectively. When comparing the CRP and MBL concentrations within the three AER categories separately, there were no significant differences between those with and without NSAID or statin treatment.

Progression The mean follow-up time was $5.8 \pm 2.2$ years, and during this period progression to either the next albuminuria level or ESRD occurred in 201 patients (Table 2). Baseline serum MBL levels were higher in progressors compared with non-progressors at all steps, but significantly so only in patients progressing from macroalbuminuria to ESRD compared with patients who remained macroalbuminuric (Table 2). In Kaplan-Meier estimates of progression made after dividing the patients into those with MBL levels above or below the median (Fig. 1a-c), the rate of progression from macroalbuminuria to ESRD was significantly higher among patients with high MBL ( $p=0.03, \log$ rank test; Fig. 1c). High MBL levels remained significantly associated with progression from macroalbuminuria to ESRD after adjustment for other significant covariates $\left(\mathrm{HbA}_{1 \mathrm{c}}\right.$, systolic blood pressure, triacylglycerol level and total cholesterol level) in a multivariate Cox regression analysis (HR 1.65, 95\% CI $1.02-2.67, p=0.040)$. The association between high MBL and progression remained the same when adding AER to the Cox analysis (HR 1.88, 95\% CI 1.06-3.32, $p=0.030$ ). Higher hsCRP levels were seen in patients that progressed from normoalbuminuria to microalbuminuria compared with patients who remained normoalbuminuric $(p=0.018)$ but not in other progressors. In a univariate analysis, the rate of progression from normoalbuminuria to microalbuminuria was significantly higher in patients with hsCRP levels above the median ( $p=0.001, \log$ rank test; Fig. 2), 


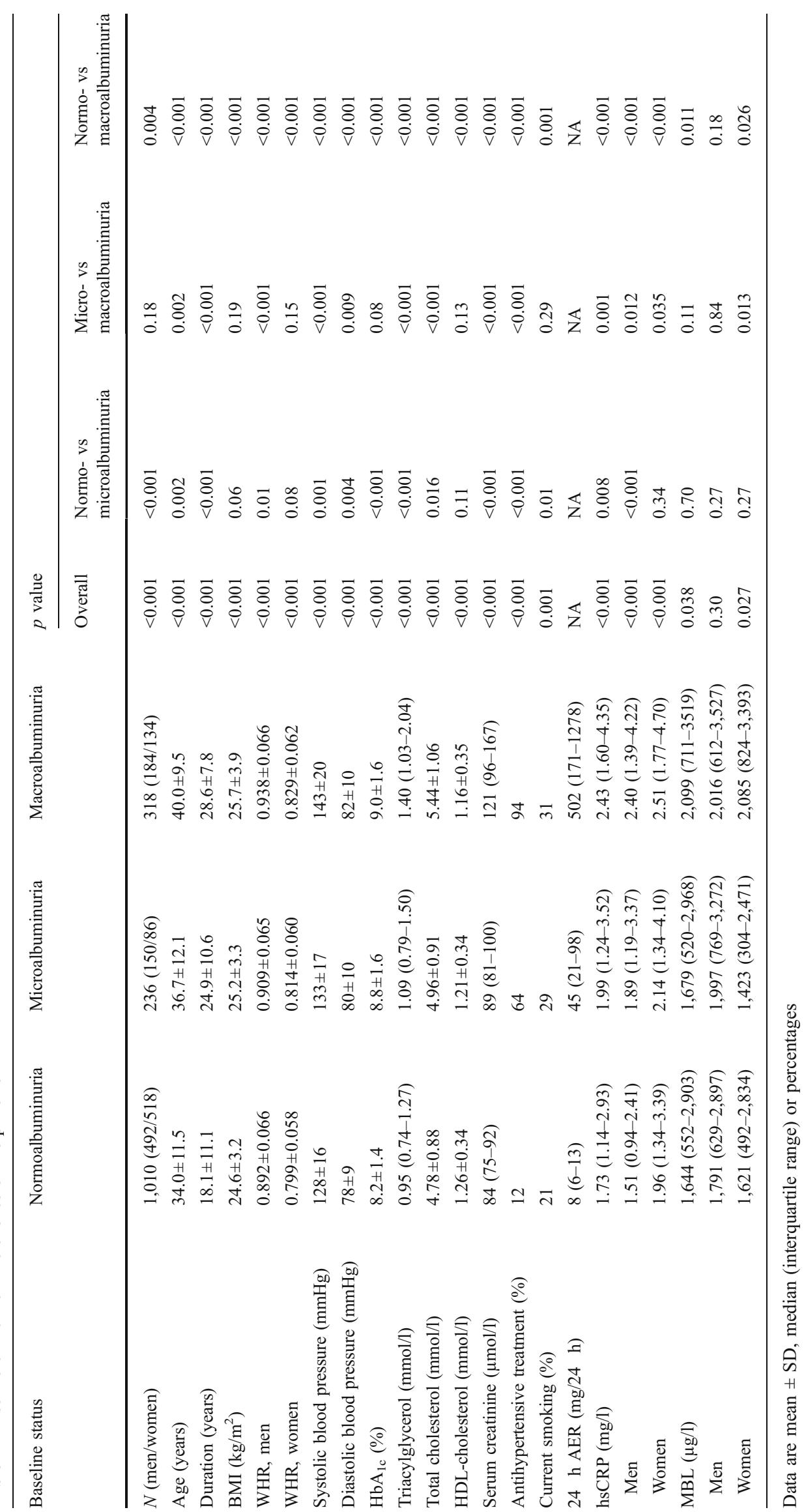




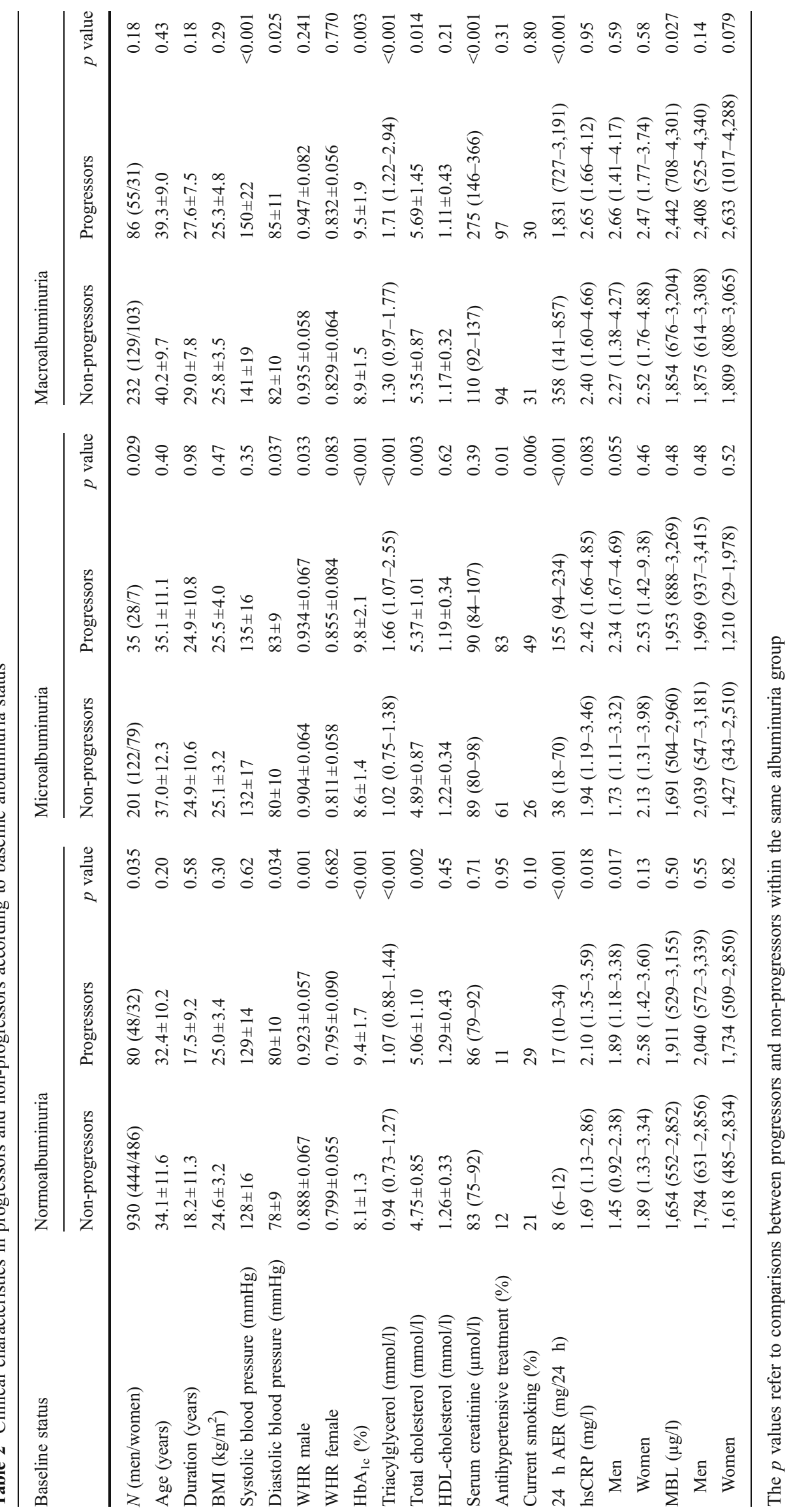



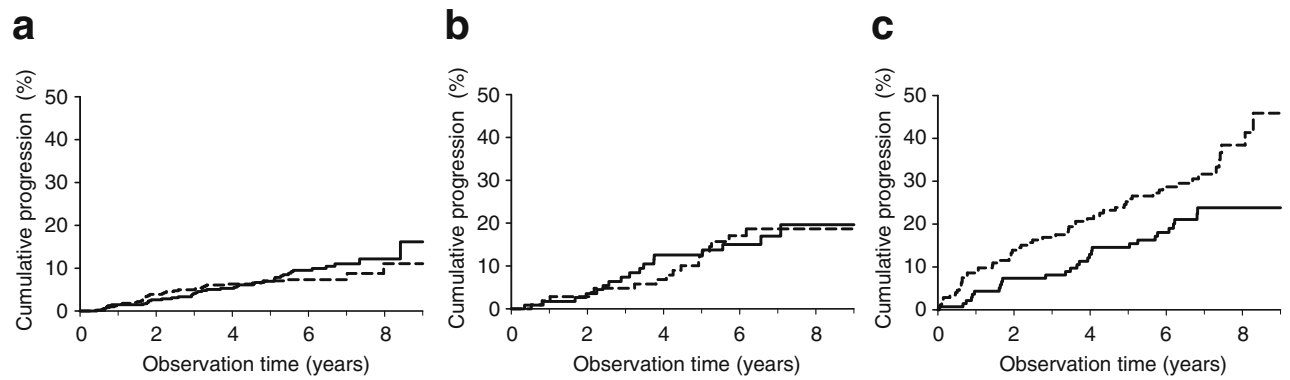

Fig. 1 Cumulative incidence of progression (a) from normo- to microalbuminuria $(p=0.32)$, (b) from micro- to macroalbuminuria $(p=$ $0.98)$ and (c) from macroalbuminuria to end-stage renal disease $(p=$ 0.03) during follow-up according to baseline MBL levels. Solid line

but the association was only borderline significant after adjustment for other significant covariates (sex, $\mathrm{HbA}_{1 \mathrm{c}}$, diastolic blood pressure, WHR and levels of triacylglycerol and total cholesterol) in a multivariate Cox-regression analysis (HR 1.56, 95\% CI 0.97-2.51, $p=0.068$ ).

In patients with type 2 diabetes, combining MBL and hsCRP may provide additional prognostic information on the development of microalbuminuria [19]. In the present study, Kaplan-Meier estimates of progression made after dividing patients according to both MBL and hsCRP median levels showed a significantly higher progression rate from normoalbuminuria to microalbuminuria in patients with both high MBL and high hsCRP compared with patients with both low MBL and low hsCRP ( $p=$ $0.011, \log$ rank test; Fig. 3). However, the association was not statistically significant in a multivariate Cox regression analysis.

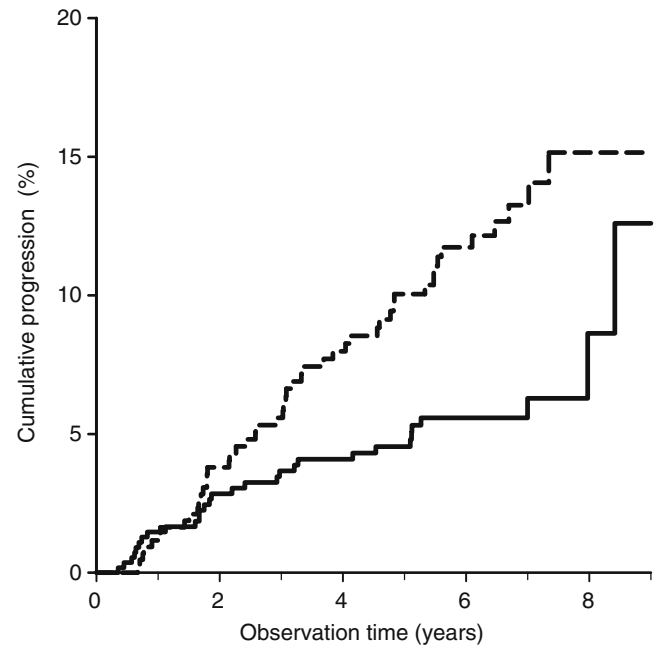

Fig. 2 Cumulative incidence of progression from normoalbuminuria to microalbuminuria during follow-up according to baseline hsCRP levels $(p=0.001$, logrank test). Solid line indicates hsCRP levels lower than the sex-specific median of $1.72 \mathrm{mg} / \mathrm{l}$ in men and $2.10 \mathrm{mg} / \mathrm{l}$ in women. Dashed line indicates hsCRP levels above the sex-specific median indicates MBL levels lower than the sex-specific median of $1,864 \mu \mathrm{g} / 1$ in men and $1,643 \mu \mathrm{g} / \mathrm{l}$ in women. Dashed line indicates MBL levels above the sex-specific medians. $p$ values refer to the logrank test

\section{Discussion}

This large prospective multicentre study confirms previous cross-sectional findings of successively increased concentrations of MBL and hsCRP in patients with increasing severity of diabetic nephropathy. In addition, the study demonstrates that both molecules are associated with the progression of renal disease in type 1 diabetes. Our findings are in line with previous studies on the associations of both MBL and hsCRP with progression of diabetic nephropathy in type 2 diabetes $[19,22]$, and points to a role of complement activation and low-grade inflammation in the pathology of diabetic kidney disease.

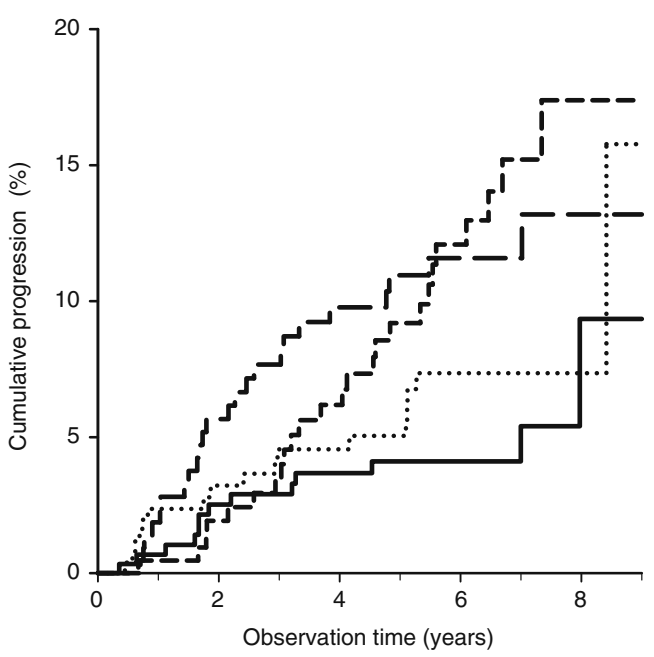

Fig. 3 Cumulative incidence of progression from normoalbuminuria to microalbuminuria during follow-up according to both baseline MBL and hsCRP levels ( $p=0.011$, logrank test). Solid line indicates MBL and hsCRP levels below the sex-specific median of 1,864 $\mu \mathrm{g} / 1$ in men and 1,643 $\mu \mathrm{g} / 1$ in women for MBL and $1.72 \mathrm{mg} / \mathrm{l}$ in men and $2.10 \mathrm{mg} / \mathrm{l}$ in women for hsCRP. Line with long dashes indicates MBL levels below and hsCRP levels above the sex-specific medians. Dotted line indicates MBL levels above and hsCRP levels below the sexspecific medians. Line with short dashes indicates MBL and hsCRP levels above the sex-specific medians 
The major part of inter-individual variation in MBL levels is genetically determined [7], and the fact that patients with diabetic nephropathy have higher levels than normoalbuminuric patients may thus point to a causative role of MBL. Assuming that patients born with high MBL levels are at increased risk of developing nephropathy, a higher proportion of these patients would have some degree of albuminuria already at baseline, which could explain why MBL levels were only associated with late progression from macroalbuminuria to ESRD in the present study. High-sensitivity CRP, by contrast, seems more associated with the earlier stages of progression, and may rather be a marker of progression than a causative factor. In epidemiological studies elevated hsCRP levels are consistently associated with atherosclerosis and vascular disease [23], and measurements of CRP levels have been used for therapeutic decision making in the primary prevention of cardiovascular events [24]. Polymorphisms in the CRP gene are associated with differences in circulating CRP levels [25], but it was recently demonstrated that these polymorphisms in themselves are not associated with an increased risk of ischaemic vascular disease [26]. Based on these findings it seems likely that CRP is merely a marker for the development of vascular disease, and one may speculate that the same is the case with respect to the progression of diabetic nephropathy.

Concentrations of MBL and hsCRP were not correlated in the present study or in previous studies of patients with type 1 diabetes mellitus $[10,12,13]$, probably as a consequence of the strong genetic influence on MBL levels. One could therefore expect a stronger association with the progression of nephropathy if prognostic information from the two variables were combined. Among normoalbuminuric patients with type 2 diabetes, those with both high MBL and high hsCRP levels have a more than twofold greater risk of progression to microalbuminuria compared with patients with both low MBL and hsCRP [22], but in the present study the combined variable was only significantly associated with the progression of nephropathy in the univariate analysis.

Our study has some limitations. First, only samples from baseline were included, and the study does not provide information on changes over time in the concentrations of the two biomarkers. For the purpose of prognostication and possible clinical use, however, the single-sample approach seems most feasible and, with respect to MBL, the day-to-day variation [27] and changes during acute phase responses [28] are very small compared with the genetically determined person-to-person variation. Second, the prospective part of the study may have been biased by the duration of diabetes at baseline. Ideally, studies of prognostic biomarkers should include patients with newly diagnosed diabetes, but in our study the average duration was approximately 20 years, and at this stage of disease patients with the highest inherent disposition are likely to have progressed to some degree of albuminuria. This is reflected in the progression rate from normo- to microalbuminuria, which was below $10 \%$ during follow-up, compared with successively higher progression rates from the more advanced stages of nephropathy.

In summary, MBL and hsCRP levels are cross-sectionally associated with the severity of diabetic nephropathy in type 1 diabetes, and both molecules may contribute information in the assessment of risk of future progression, albeit with different strengths and at different stages of renal disease.

Acknowledgements This study was supported by the Danish Medical Research Council, The Danish Diabetes Association, Clinical Institute, Aarhus University Hospital, the Novo Nordisk Foundation, and the Danielsen Foundation. The FinnDiane Study was supported by grants from the Folkhälsan Research Foundation, Wilhelm and Else Stockmann Foundation, Liv och Hälsa Foundation, Finnish Medical Society (Finska Läkaresällskapet), Waldemar von Frenckell Foundation, Sigrid Juselius Foundation, Governmental Grants for Health Sciences Research (EVO) and the European Commission (QLG2-CT-2001-01669, LSHB-CT-2003-503364, LSHB-CT-2006037681). We gratefully acknowledge the skilful laboratory assistance of D. E. Wulff, A. Sandelin, M. Parkkonen, A.-R. Salonen and J. Tuomikangas. We also acknowledge all the physicians and nurses at each centre participating in the collection of patients, details of whom are presented in the Electronic supplementary material (ESM 1).

Duality of interest The authors declare that there is no duality of interest associated with this manuscript.

\section{References}

1. Hovind P, Tarnow L, Rossing P et al (2004) Predictors for the development of microalbuminuria and macroalbuminuria in patients with type 1 diabetes: inception cohort study. BMJ 328:1105

2. Walport MJ (2001) Complement. First of two parts. N Engl J Med 344:1058-1066

3. Walport MJ (2001) Complement. Second of two parts. N Engl J Med 344:1140-1144

4. Qin X, Goldfine A, Krumrei N et al (2004) Glycation inactivation of the complement regulatory protein CD59: a possible role in the pathogenesis of the vascular complications of human diabetes. Diabetes 53:2653-2661

5. Uesugi N, Sakata N, Nangaku M et al (2004) Possible mechanism for medial smooth muscle cell injury in diabetic nephropathy: glycoxidation-mediated local complement activation. Am J Kidney Dis 44:224-238

6. Ostergaard J, Thiel S, Gadjeva M, Hansen TK, Rasch R, Flyvbjerg A (2007) Mannose-binding lectin deficiency attenuates renal changes in a streptozotocin-induced model of type 1 diabetes in mice. Diabetologia 50:1541-1549

7. Best LG, Ferrell RE, DeCroo S et al (2009) Genetic and other factors determining mannose-binding lectin levels in American Indians: the Strong Heart Study. BMC Med Genet 10:5

8. Kaunisto M, Sjolind L, Sallinen R et al (2009) Elevated MBL concentrations are not an indication of association between the MBL2 gene and type 1 diabetes or diabetic nephropathy. Diabetes 58:1710-1714 
9. Aittoniemi J, Turpeinen H, Tiittanen M et al (2008) Relation among mannose-binding lectin 2 genotype, beta-cell autoantibodies, and risk for type 1 diabetes in Finnish children. Hum Immunol 69:108-111

10. Hansen TK, Thiel S, Knudsen ST et al (2003) Elevated levels of mannan-binding lectin in patients with type 1 diabetes. J Clin Endocrinol Metab 88:4857-4861

11. Bouwman LH, Eerligh P, Terpstra OT et al (2005) Elevated levels of mannose-binding lectin at clinical manifestation of type 1 diabetes in juveniles. Diabetes 54:3002-3006

12. Hansen TK, Tarnow L, Thiel S et al (2004) Association between mannose-binding lectin and vascular complications in type 1 diabetes. Diabetes 53:1570-1576

13. Saraheimo M, Forsblom C, Hansen TK et al (2005) Increased levels of mannan-binding lectin in type 1 diabetic patients with incipient and overt nephropathy. Diabetologia 48:198-202

14. Hovind P, Hansen TK, Tarnow L et al (2005) Mannose-binding lectin as a predictor of microalbuminuria in type 1 diabetes: an inception cohort study. Diabetes 54:1523-1527

15. Schalkwijk CG, Poland DC, van Dijk W et al (1999) Plasma concentration of C-reactive protein is increased in type I diabetic patients without clinical macroangiopathy and correlates with markers of endothelial dysfunction: evidence for chronic inflammation. Diabetologia 42:351-357

16. Jenkins AJ, Rothen M, Klein RL et al (2008) Cross-sectional associations of C-reactive protein with vascular risk factors and vascular complications in the DCCT/EDIC cohort. J Diabetes Complicat 22:153-163

17. Saraheimo M, Teppo AM, Forsblom C, Fagerudd J, Groop PH (2003) Diabetic nephropathy is associated with low-grade inflammation in type 1 diabetic patients. Diabetologia 46:1402-1407

18. Schaumberg DA, Glynn RJ, Jenkins AJ et al (2005) Effect of intensive glycemic control on levels of markers of inflammation in type 1 diabetes mellitus in the diabetes control and complications trial. Circulation 111:2446-2453
19. Stehouwer CD, Gall MA, Twisk JW, Knudsen E, Emeis JJ, Parving HH (2002) Increased urinary albumin excretion, endothelial dysfunction, and chronic low-grade inflammation in type 2 diabetes: progressive, interrelated, and independently associated with risk of death. Diabetes 51:1157-1165

20. Retnakaran R, Cull CA, Thorne KI, Adler AI, Holman RR (2006) Risk factors for renal dysfunction in type 2 diabetes: U.K. Prospective Diabetes Study 74. Diabetes 55:1832-1839

21. Lin J, Glynn RJ, Rifai N et al (2008) Inflammation and progressive nephropathy in type 1 diabetes in the diabetes control and complications trial. Diabetes Care 31:2338-2343

22. Hansen TK, Gall MA, Tarnow L et al (2006) Mannose-binding lectin and mortality in type 2 diabetes. Arch Intern Med 166: 2007-2013

23. Verma S, Szmitko PE, Ridker PM (2005) C-reactive protein comes of age. Nat Clin Pract Cardiovasc Med 2:29-36

24. Ridker PM, Danielson E, Fonseca FA et al (2008) Rosuvastatin to prevent vascular events in men and women with elevated Creactive protein. N Engl J Med 359:2195-2207

25. Hage FG, Szalai AJ (2007) C-reactive protein gene polymorphisms, C-reactive protein blood levels, and cardiovascular disease risk. J Am Coll Cardiol 50:1115-1122

26. Zacho J, Tybjaerg-Hansen A, Jensen JS, Grande P, Sillesen H, Nordestgaard BG (2008) Genetically elevated C-reactive protein and ischemic vascular disease. N Engl J Med 359:1897-1908

27. Hansen TK, Thiel S, Dall R et al (2001) GH strongly affects serum concentrations of mannan-binding lectin: evidence for a new IGF-I independent immunomodulatory effect of GH. J Clin Endocrinol Metab 86:5383-5388

28. Hansen TK, Thiel S, Wouters PJ, Christiansen JS, van den Berghe G (2003) Intensive insulin therapy exerts antiinflammatory effects in critically ill patients and counteracts the adverse effect of low mannose-binding lectin levels. J Clin Endocrinol Metab 88:10821088 